\title{
Exploring the Use of Type-2 Fuzzy Sets in Multi-criteria Decision Making based on TOPSIS
}

\author{
Elissa Nadia Madi ${ }^{1}$, Jonathan M. Garibaldi ${ }^{1}$, Christian Wagner ${ }^{2}, 1$ \\ ${ }^{1}$ Lab for Uncertainty in Data and Decision Making (LUCID) and IMA Research Group, \\ School of Computer Science, University of Nottingham, UK \\ ${ }^{2}$ Institute of Computing and Cybersystems (ICC), Michigan Technological University, USA. \\ \{elissa.madi,jon.garibaldi,christian.wagner\}@nottingham.ac.uk
}

\begin{abstract}
Multi-criteria decision making (MCDM) problems are a well known category of decision making problem that has received much attention in the literature, with a key approach being the Technique for Order Preference by Similarity to Ideal Solution (TOPSIS). While TOPSIS has been developed towards the use of Type-2 Fuzzy Sets (T2FS), to date, the additional information provided by T2FSs in TOPSIS has been largely ignored since the final output, the Closeness Coefficient (CC), has remained a crisp value. In this paper, we develop an alternative approach to $\mathrm{T} 2$ fuzzy TOPSIS, where the final $\mathrm{CC}$ values adopt an interval-valued form. We show in a series of systematically designed experiments, how increasing uncertainty in the T2 membership functions affects the interval-valued $\mathrm{CC}$ outputs. Specifically, we highlight the complex behaviour in terms of the relationship of the uncertainty levels and the outputs, including non-symmetric and non-linear growth in the $\mathrm{CC}$ intervals in response to linearly growing levels of uncertainty. As the first TOPSIS approach which provides an interval-valued output to capture output uncertainty, the proposed method is designed to reduce the loss of information and to maximize the benefit of using T2FSs. The initial results indicate substantial potential in the further development and exploration of the proposed and similar approaches and the paper highlights promising next steps.
\end{abstract}

\section{INTRODUCTION}

Multi-criteria decision making (MCDM) problems are a well known category of decision making problem, and have been the focus of much research effort. This problem concerns finding the most desirable alternative(s) from a set of predetermined alternatives, $A=\left\{A_{1}, A_{2}, \cdots, A_{n}\right\}$ with respect to the decision information about criteria weights and criteria values provided by a group of Decision Makers (DMs), $D M=\left\{D M_{1}, D M_{2}, \cdots, D M_{m}\right\}$. However, a major problem with dealing with human decision makers is that they exhibit variation in their decision making [6]. In order to design a truly intelligent decision support model, such variations should be considered, especially at the beginning of the process itself.

Fuzzy MCDM methods, provide the means to assign linguistic labels (e.g., Very Good, Very Poor, etc.) that use membership functions (MFs) to represent the performance of each alternative with respect to each criterion. For example, a well-known MCDM technique is the 'Fuzzy Technique for Order Preference by Similarity to Ideal Solution' (FTOPSIS) introduced by Chen in 2000 [2]. In this method, the performance of each alternative is evaluated against each criterion using a scale made up of fuzzy MFs. Such sets are used to enhance pre-screening evaluations; where the value of a positive performance rating (e.g. Good), can be captured in a range of values from 5 to 10 . Then, an experimental setup is modeled using fuzzy set methods.

A wide variety of fuzzy MCDM applications have been developed over the last two decades. Behzadian et al. [1] reviewed applications of TOPSIS and found that using fuzzy sets is the most commonly used method in TOPSIS. Specifically, while the classical TOPSIS method [7] assumes that alternative ratings and criteria weights are crisp numbers, more than half of the TOPSIS publications (52.2\%) utilize linguistic variables and fuzzy sets to handle problems with imprecise information. An initial extension of the TOPSIS method for group decision-making under a fuzzy environment by Chen [2] in 2000 is one of the early works in this area. Following this work, many researchers have started to explore this area and to propose further developments. Various types of enhancements have been made, including implementations of Type-2 (T2) fuzzy sets in TOPSIS methods. Notable progress was made in a method proposed by Chen and Lee [3], in which Interval Type-2 (IT2) fuzzy sets are used with TOPSIS. The authors determined ranking values using elements in a fuzzy decision matrix and from a crisp ranking, they then calculated the Closeness Coefficient (CC) values using both methods and compared the results.

More recently, Sang and Liu [12] highlighted the potential of information loss in respect to the decision matrix when applying ranking [3], as defuzzification takes place in the beginning. They proposed an alternative where IT2FSs are preserved throughout the process. Other researchers, however, have focused on specific steps of the TOPSIS method. For example, in [11], the authors propose an IT2 fuzzy TOPSIS approach in which specifically the determination of a fuzzy positive ideal solution and a fuzzy negative ideal solution are achieved using IT2 fuzzy sets. Recently, Dymova et. al. [4], proposed an IT2 fuzzy TOPSIS method using alpha cuts, and Kilic and Kaya [8], proposed an IT2 fuzzy TOPSIS method to evaluate an investment project producing a final ranking.

Clearly, in all the studies reviewed here, IT2 fuzzy TOPSIS is recognized as a useful method of addressing MCDM problems. However, the additional information provided by IT2 FSs in these methods reviewed here is largely lost, since the final output (the $\mathrm{CC}$ ) is still a crisp value. Thus, the 
benefit of using such sets is not fully exploited, as the extra information that they could provide is not available to DMs in the decision making process. Hence, it is intuitive to develop a TOPSIS method based on IT2 FSs which takes advantage of the additional information provided by the sets, where the final result remains an interval. Motivated by this, we conduct an initial set of experiments that introduces a number of different levels of uncertainty using IT2 MFs that are associated with linguistic labels (e.g. Good, Fair, etc.) that are produced by blurring initial T1 MFs. The method used in the experiment preserves the additional uncertainty of the final output (CC) by maintaining its interval form. The interval result of the proposed method is the key difference between our method and the standard fuzzy TOPSIS methods that exist, which produce a crisp CC. By conducting the experiments we specifically explore whether any (and which) relationship exists between the level of uncertainty (amount of blurring) and the TOPSIS result, i.e. the $\mathrm{CC}$ interval. To date, no other researchers have proposed a fuzzy TOPSIS method which provides an interval as a final output allowing a richer basis for ranking. In future research we intend to further explore the associated benefits in terms of decision making provided by T2 FSs further.

The paper is structured as follows: In Section II a detailed discussion about the key stages in standard TOPSIS and fuzzy TOPSIS is provided. Section III presents the methodology used in this paper as well as an example to show how each stage works. Section IV briefly explains the experimental procedure used in this study. Section $\mathrm{V}$ provides a detailed discussion on the experimental results and findings, and finally Section VI presents the conclusions we have drawn from this work and potential paths of future work.

\section{BACKGROUND}

\section{A. The TOPSIS method}

In MCDM techniques, there are two basic approaches: multiple attribute decision making (MADM) and multiple objective decision making (MODM) [8]. TOPSIS falls in the MADM category which refers to making a selection from a number of courses of action in the presence of multiple, usually conflicting, attributes. The standard TOPSIS method is to choose alternatives that simultaneously have the shortest distance from the positive ideal solution (PIS) (hypothetical best alternative) and the farthest distance from the negativeideal solution (NIS) (hypothetical worst alternative) [7]. In the standard TOPSIS method, the ratings and weights of criteria are known precisely and crisp data is used to model realworld situations. Such ratings and weights of criteria can be concisely expressed in decision matrix format as:

$$
\begin{aligned}
& \begin{array}{llll}
C_{1} & C_{2} & \ldots & C_{n}
\end{array} \\
& \mathbf{D}=\begin{array}{l}
A_{1} \\
A_{2} \\
\vdots \\
A_{m}
\end{array}\left[\begin{array}{cccc}
x_{11} & x_{12} & \cdots & x_{1 n} \\
x_{21} & x_{22} & \cdots & x_{2 n} \\
\vdots & \vdots & \ddots & \vdots \\
x_{m 1} & x_{m 2} & \cdots & x_{m n}
\end{array}\right], \\
& \mathbf{W}=\left[\begin{array}{llll}
w_{1} & w_{2} & \cdots & w_{n}
\end{array}\right],
\end{aligned}
$$

where $A_{1}, A_{2}, \cdots, A_{m}$ are possible alternatives among which DMs have to choose, $C_{1}, C_{2}, \cdots, C_{n}$ are criteria/attributes with which measured, $x_{i j}$ is the rating of alternative $A_{i}$ with respect to criterion $C_{j}$ and $w_{j}$ is the weight of criterion $C_{j}$.

In standard TOPSIS, it is assumed that each attribute in the decision matrix $\mathbf{D}$ captures either monotonically increasing or monotonically decreasing utility. In other words, the larger the attribute outcome is, the greater the preference for the 'benefit' criteria and the lower the preference for the 'cost' criteria. Furthermore, any outcome expressed in a non-numerical format should be converted based on an appropriate scaling technique, for example, using a rating scale on certain points on $[0,10]$ or $[0,100]$ by a group of DMs. Since all criteria are commonly not of equal importance, the method uses a set of weights from the DMs, captured in a matrix $\mathbf{W}$ using the weight of importance scale. In general, it is often impractical to use crisp/numeric assessments for modeling in such situations. For example, human judgments or preferences are often vague and it is not possible to capture such complex preferences in an exact numerical form. Thus, fuzzy sets can be used to express preferences using linguistic variables. The general Fuzzy TOPSIS (FTOPSIS) method has been a popular choice in the MCDM field over the last 15 years [2], and will be reviewed in the next section.

\section{B. The Fuzzy TOPSIS (FTOPSIS) method}

We provide the step-wise procedure of the standard FTOPSIS method [2] [10] in Fig. 1. In FTOPSIS, a special type of fuzzy set, namely a fuzzy number, is used to represent human preferences. A fuzzy number is a fuzzy set in the universe of discourse $X$ that is both convex and normal. There are different types of fuzzy numbers, the Triangular Fuzzy Number (TFN) is most widely used in decision making because of its intuitive membership functions and computational simplicity [5] [9]. A TFN can be defined as a triplet $(l, m, u)$, where the parameters $l$ and $u$ specify the start and end of the membership function, while $m$ is the point where the membership function peaks.

At the start of FTOPSIS, the same matrices as in standard TOPSIS are used to present the rating of alternatives and the weights of criteria, namely the decision matrices $\mathbf{D}$ and $\mathbf{W}$, (see Section II-A). However, instead of using crisp numbers in the ratings of $x_{i j}$, the FTOPSIS method uses fuzzy numbers (FN) to represent them. For example, for rating Good, the associated triangular FN is $(7,9,10)$ as shown in Table I. In contrast, in standard TOPSIS, the value of Good might be the crisp value 9. Assume that a decision group consists of $k$ decision makers, then the average weights of criteria and ratings of alternatives given by $k$ decision makers can be computed as:

$$
\begin{gathered}
\tilde{w}_{j}=\frac{1}{k}\left[\tilde{w}_{j}^{1}+\tilde{w}_{j}^{2}+\cdots+\tilde{w}_{j}^{k}\right], \\
\tilde{x}_{j}=\frac{1}{k}\left[\tilde{x}_{j}^{1}+\tilde{x}_{j}^{2}+\cdots+\tilde{x}_{j}^{k}\right],
\end{gathered}
$$

where $\tilde{w}_{j}$ and $\tilde{x}_{j}$ are the importance weights of criteria and the ratings of alternatives given by $k$ th decision maker. The 


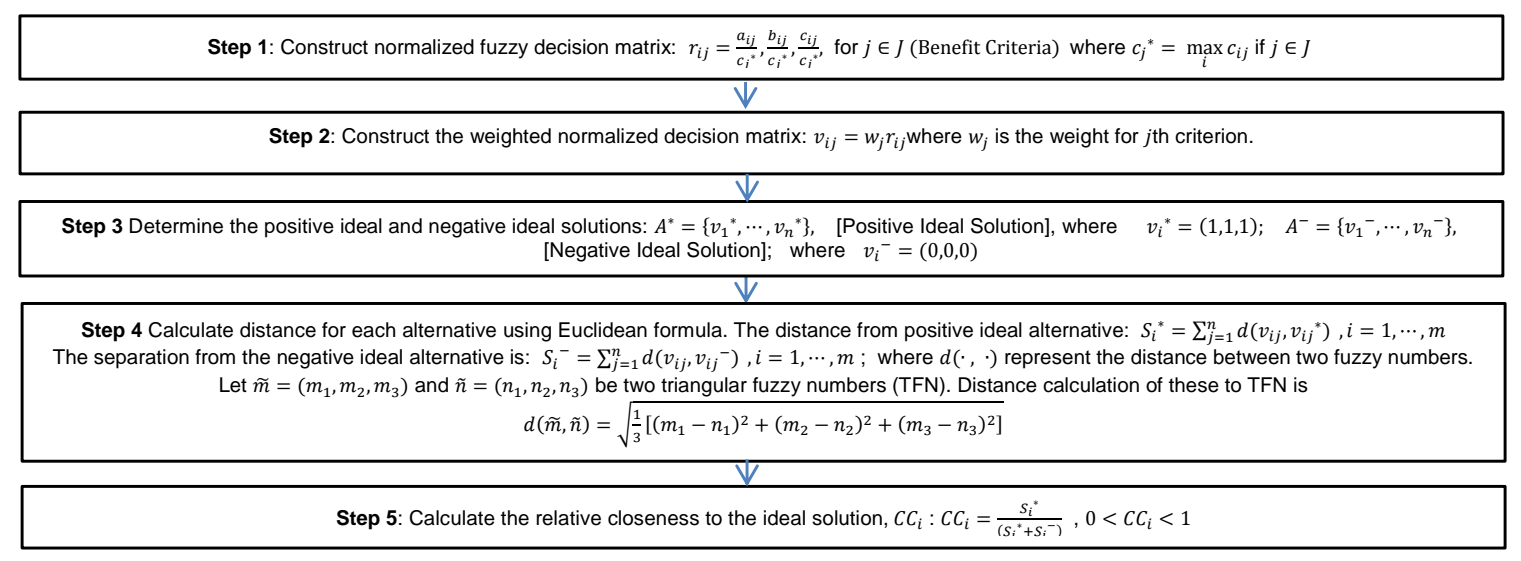

Fig. 1. Step-wise procedure of Fuzzy TOPSIS [2], [10]

resulting values of $\tilde{w}_{j}$ and $\tilde{x}_{j}$ are expressed in the matrices $\mathbf{W}$ and $\mathbf{D}$, respectively. The ratings and the weights are described as triangular fuzzy numbers which indicate $\tilde{x}_{j}=\left(a_{i j}, b_{i j}, c_{i j}\right)$ and $\tilde{w}_{j}=\left(w_{j 1}, w_{j 2}, w_{j 3}\right)$. Then, the stepwise procedure of FTOPSIS proceeds as shown in Fig. 1.

In Step 1, the fuzzy number for its associated membership function is expressed as $\left(a_{i j}, b_{i j}, c_{i j}\right)$, where $a, b$ and $c$ are the smallest possible value, the peak value and the largest possible value, respectively. The normalization step is needed to transform various criteria scales into a single comparable scale, and to maintain the FNs in $[0,1]$. In this step, $J$ is associated with the benefit criteria. For Step 2, a set of weights expressed in $\mathbf{W}$ given by DMs are applied to the decision matrix, $\mathbf{D}$ by multiplying each column of the matrix $\mathbf{D}$ (i.e., $r_{i j}$ value) with its associated weight $w_{j}$. Then, in Step 3, two artificial alternatives known as Positive Ideal Solution (PIS), $A^{*}$, and Negative Ideal Solution (NIS), $A^{-}$, are defined. Step 4 is a calculation separation between each alternative from the ideal one by the $n$-dimensional Euclidean distance. Then, in the final step, the relative Closeness Coefficient $\left(C C_{i}\right)$ of alternatives $i$ th $\left(A_{i}\right)$ with respect to $A^{*}$ is calculated. It is clear that $C C_{i}=1$ if $A_{i}=A^{*}$ and $C C_{i}=0$ if $A_{i}=A^{-}$. An alternative $A_{i}$ is closer to $A^{*}$ as $C C_{i}$ approaches 1 . Next, an alternative can now be ranked according to the descending order of $C C_{i}$. An example of these steps being used in a decision problem is presented in Section III.

TABLE I

LINGUISTIC SCALE FOR WEIGHTING OF CRITERIA AND RATING OF ALTERNATIVES IN THE FUZZY TOPSIS METHOD [2]

\begin{tabular}{|l|c|l|c|}
\hline \multicolumn{2}{|c|}{ (a) Rating scale } & \multicolumn{2}{c|}{ (b)Weighting scale } \\
\hline Very Poor (VP) & $(0,0,1)$ & Very Low & $(0,0,0.1)$ \\
Poor (P) & $(0,1,3)$ & Low (L) & $(0,0.1,0.3)$ \\
Medium Poor (MP) & $(1,3,5)$ & Medium Low (ML) & $(0.1,0.3,0.5)$ \\
Fair (F) & $(3,5,7)$ & Medium (M) & $(0.3,0.5,0.7)$ \\
Medium Good (MG) & $(5,7,9)$ & Medium High (MH) & $(0.5,0.7,0.9)$ \\
Good (G) & $(7,9,10)$ & High (H) & $(0.7,0.9,1.0)$ \\
Very Good (VG) & $(9,10,10)$ & Very High (VH) & $(0.9,1.0,1.0)$ \\
\hline
\end{tabular}

TABLE II

RATINGS OF ALL APPLICANTS (ALTERNATIVES) AND IMPORTANCE WEIGHTS OF CRITERIA GIVEN BY DECISION MAKERS

\begin{tabular}{|c|c|c|c|c|c|c|c|}
\hline & & $\mathrm{DM}^{\prime}$ & 's rat & & & & DM's rating \\
\hline Crit. & Alts. & $D_{1}$ & $D_{2}$ & $D_{3}$ & Crit. & Alts. & $\begin{array}{lll}\text { D1 } & \text { D2 } & \text { D3 }\end{array}$ \\
\hline \multirow[t]{3}{*}{$C_{1}$} & $A_{1}$ & MG & $\mathrm{G}$ & MG & $C_{4}$ & $A_{1}$ & VG $\mathrm{G}$ \\
\hline & $A_{2}$ & & & MG & & $A_{2}$ & VG VG VG \\
\hline & $A_{3}$ & MG & MG & $\mathrm{F}$ & & $A_{3}$ & $\mathrm{G} \quad \mathrm{VG} \quad \mathrm{MG}$ \\
\hline \multicolumn{2}{|c|}{ Weight } & VH & VH & VH & \multicolumn{2}{|c|}{ Weight } & VH VH VH \\
\hline \multirow[t]{3}{*}{$C_{2}$} & $A_{1}$ & $\mathrm{G}$ & MG & $\mathrm{F}$ & $C_{5}$ & $A_{1}$ & $\begin{array}{lll}\mathrm{F} & \mathrm{F} & \mathrm{F}\end{array}$ \\
\hline & $A_{2}$ & & G & G & & $A_{2}$ & VG $\mathrm{MG} \mathrm{G}$ \\
\hline & $A_{3}$ & MG & G & $\mathrm{G}$ & & $A_{3}$ & $\mathrm{G} \quad \mathrm{G} \quad \mathrm{MG}$ \\
\hline \multicolumn{2}{|c|}{ Weight } & $\mathrm{M}$ & $\mathrm{MH}$ & MH & \multicolumn{2}{|c|}{ Weight } & VH VH VH \\
\hline \multirow[t]{3}{*}{$C_{3}$} & $A_{1}$ & & MG & MG & & & \\
\hline & $A_{2}$ & & & MG & & & \\
\hline & $A_{3}$ & & MG & MG & & & \\
\hline \multicolumn{2}{|c|}{ Weight } & VH & $\mathrm{H}$ & $\mathrm{H}$ & & & \\
\hline
\end{tabular}

\section{Methodology}

\section{Example: Analyzing the steps of the FTOPSIS method}

In order to show how each stage in the standard FTOPSIS method shown in Fig. 1 works, we provide a practical stepby-step walk-through based on the example in [2]. Suppose that a software company wants to hire a system analysis engineer. After preliminary screening, three applicants $A_{1}, A_{2}$ and $A_{3}$ are retained for further evaluation. A committee of three decision-makers, $D_{1}, D_{2}$ and $D_{3}$ has been formed to conduct the interview and to select the most suitable applicant. Five desirable criteria are considered; emotional steadiness $\left(C_{1}\right)$; oral communication skills $\left(C_{2}\right)$, personality $\left(C_{3}\right)$, past experience $\left(C_{4}\right)$ and self-confidence $\left(C_{5}\right)$. The DMs use the linguistic scale in Table I to give ratings and weightings which are presented in Table II.

Having rated the alternatives, the next step is to aggregate all the evaluated values to form a fuzzy decision matrix using Eq. (2). Next, a fuzzy decision matrix of the alternatives is constructed (i.e. D), to show all of the subjective ratings given by the set of decision makers. Post aggregation, a normalization step (Step 1 in Fig. 1) is applied. For this example, the results of this process are shown in Table 


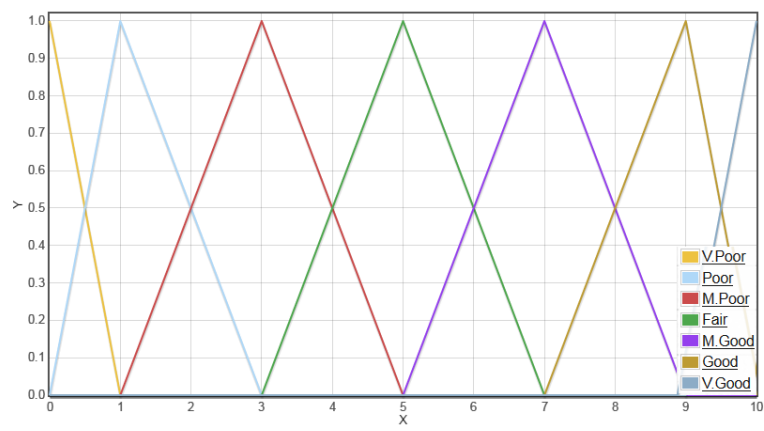

Fig. 2. Triangular Fuzzy Numbers for rating scale

III. The next step is to define the fuzzy PIS and NIS in this case, the perfect values are used. The fuzzy PIS is defined as: $A^{+}=[(1,1,1),(1,1,1),(1,1,1),(1,1,1),(1,1,1)]$ while the fuzzy NIS is defined as: $A^{-}=[(0,0,0),(0,0,0),(0,0,0),(0,0,0),(0,0,0)]$. These values are the result of Step 3 in Fig. 1. The next step is to find the distance between the ideal solution and each of the alternatives. This is done using the method described in the fourth step of Fig. 1. In this example, the CC values (Step 5) and rankings of alternatives are summarized as follows: $C C\left(A_{1}\right)=0.6296, \operatorname{rank}_{A 1}=3, C C\left(A_{2}\right)=0.7374$, $\operatorname{rank}_{A 2}=1$ and $C C\left(A_{3}\right)=0.7059, \operatorname{rank}_{A 3}=2$. In this case, alternative $A_{2}$ is the best, followed by $A_{3}$ and $A_{1}$. From this result, in the standard Fuzzy TOPSIS approach considered here, the $\mathrm{CC}$ values are calculated as crisp values. To get the final ranking, the values are sorted into descending order.

\section{EXPERIMENT DESIGN}

\section{A. Introduction}

In this section, we describe our experiment design. A series of small changes are introduced to the standard T1 fuzzy MFs (Fig. 2) to construct IT2 fuzzy MFs (Fig. 4), based on the TOPSIS method. These changes are driven by varying $\delta \in[0,1]$. The delta values used in this experiment are: $\delta=(0.10,0.13,0.14,0.145,0.15,0.20,0.30,0.40,0.50,0.60)$. Each delta value is used to shift the MFs used in the standard fuzzy TOPSIS method (Fig. 2) both left and right as shown in Fig. 3. The CC values that are the output of the process are in interval form, thus preserving additional information compared to crisp outputs. Thus, the uncertainty information encapsulated in the IT2 fuzzy sets and reflected in the CC intervals is available to DMs to guide their decision making process. In the next section, we provide further details on how T1 FNs are 'expanded' to T2 FNs using an example.

\section{B. Example: Fuzzy TOPSIS with $\delta=0.50$}

In order to demonstrate the proposed approach, we apply $\delta=0.50$ to T1 fuzzy numbers to produce IT2 fuzzy numbers to be used in the Fuzzy TOPSIS process. Figure 3 shows the effect of the direct expanding MFs technique on a T1 fuzzy number, here, representing the linguistic label Fair. The figure shows the original T1 MF and the resulting IT2 fuzzy number bounded by an Upper Membership Function (UMF) and a

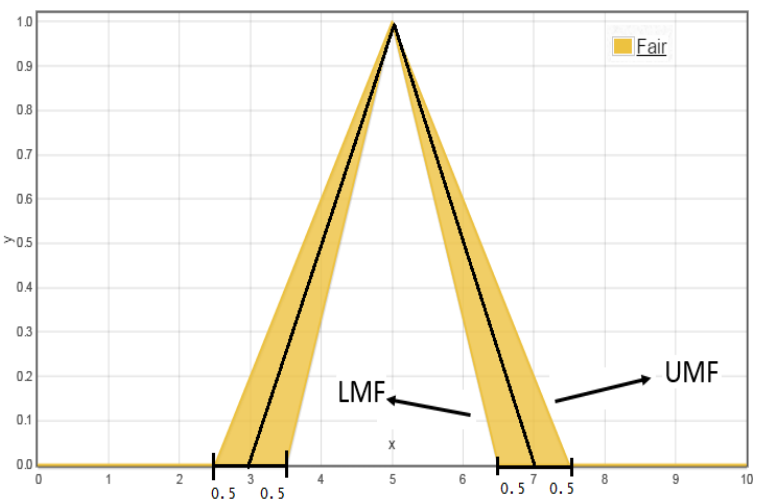

Fig. 3. Triangular IT2 FN for label Fair, blurring $\delta=0.50$

Lower Membership Function (LMF). Next, this method is repeated for the other linguistic labels in the rating scale. Each fuzzy label is defined with an associated fuzzy number value. For example, the linguistic label Fair in Fig. 3, is defined as $[(2.5,5,7.5),(3.5,5,6.5)]$, where the first and second elements capture an upper and lower values, respectively. Note that the IT2 MFs generated are based on the original T1 MFs from Fig. 2. The remaining labels for the linguistic ratings scale now become IT2 MFs as shown in Fig. 4. The new scale can be defined with IT2 fuzzy numbers as shown in Table IV, where (a) represents the upper values and (b) represents the lower values. The decision makers then use the IT2 fuzzy numbers to give ratings for each alternative.

Finally, the fuzzy TOPSIS procedure as shown in Section

TABLE III

FUZZY DECISION MATRIX

\begin{tabular}{|c|ccc|}
\hline & $C_{1}$ & $C_{2}$ & $C_{3}$ \\
\hline$A_{1}$ & $(5.67,7.67,9.33)$ & $(5.00,7.00,8.67)$ & $(5.67,7.67,9)$ \\
$A_{2}$ & $(6.33,8.33,9.67)$ & $(9,10,10)$ & $(8.33,9.67,10)$ \\
$A_{3}$ & $(6.33,8.33,9.00)$ & $(7,8.67,9.67)$ & $(7,8.67,9.67)$ \\
\hline Weight & $(0.70,0.87,0.97)$ & $(0.90,1.00,1.00)$ & $(0.77,0.93,1.00)$ \\
\hline & $C_{4}$ & $C_{5}$ \\
\hline$A_{1}$ & $(8.33,9.67,9)$ & $(3,5,7)$ \\
$A_{2}$ & $(9,10,10)$ & $(7,8.67,9.67)$ & \\
$A_{3}$ & $(7,8.67,9.67)$ & $(6.3,8.3,9.67)$ & \\
\hline Weight & $(0.90,1,1)$ & $(0.43,0.63,0.83)$ & \\
\hline
\end{tabular}

TABLE IV

INTERVAL TyPe-2 FuZzy LiNGUistic SCALE For Rating OF Alternatives WHEN $\delta=0.50$

\begin{tabular}{|l|c|c|}
\hline \multicolumn{1}{|c|}{ Rating Label } & $\begin{array}{c}\text { (a)Upper values for } \\
\text { IT2 fuzzy MFs }\end{array}$ & $\begin{array}{c}\text { (b)Lower values for } \\
\text { IT2 fuzzy MFs }\end{array}$ \\
\hline Very Poor (VP) & $(0,0,1.5)$ & $(0,0,0.5)$ \\
Poor (P) & $(-0.5,1,3.5)$ & $(0.5,1,2.5)$ \\
Medium Poor (MP) & $(0.5,3,5.5)$ & $(1.5,3,4.5)$ \\
Fair (F) & $(2.5,5,7.5)$ & $(3.5,5,6.5)$ \\
Medium Good (MG) & $(4.5,7,9.5)$ & $(5.5,7,8.5)$ \\
Good (G) & $(6.5,9,10.5)$ & $(7.5,9,9.5)$ \\
Very Good (VG) & $(8.5,10,10.5)$ & $(9.5,10,9.5)$ \\
\hline
\end{tabular}




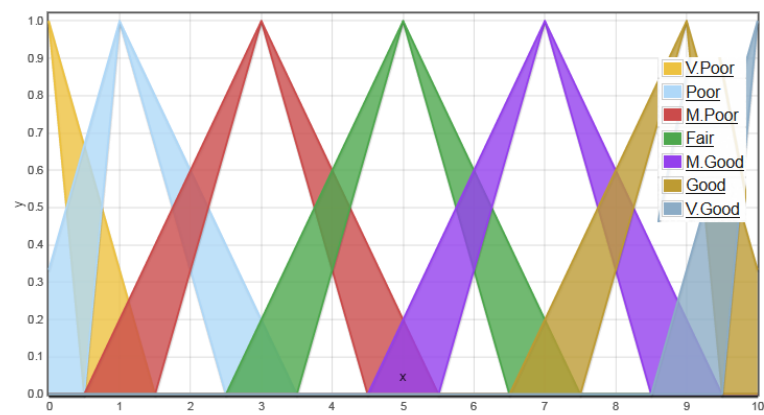

Fig. 4. Triangular FN for rating scale, blurring $\delta=0.50$

II-B is applied to get the rank of each alternative. To recap, the procedure starts by collecting the ratings from DMs and calculates the average for each rating value. The results are represented as IT2 fuzzy numbers that is captured in a decision matrix, $\mathbf{D}$, which has two elements for each rating, namely for the upper and lower values. Then, those values are processed separately in each step as described in Section II-B. Since we preserve the uncertainty (by considering the footprint of uncertainty, i.e. the upper and lower values, separately) from the start to the end of procedure, the final $\mathrm{CC}$ values can be expressed in interval form based on the two individual outputs. Note that each $\mathrm{CC}$ value indicates the relative distance in respect to PIS and NIS as shown in example in Section III. We present the results in Table $\mathrm{V}$ and Fig. 5 for all values of $\delta$.

\section{DISCUSSION}

In the experiments, for all $\delta$, the rank order of the alternatives, $A_{1}, A_{2}$ and $A_{3}$, based on the centre of each interval, remains the same as in Chen's [2] example shown in Section III, namely that $A_{2}$ is the best followed by $A_{3}$ and $A_{1}$. Figure 5 shows the results of the IT2 fuzzy TOPSIS inference methodology based on each of the delta values (uncertainty levels) used in this experiment. All previous studies of IT2 fuzzy TOPSIS have focused only on the centre-point of the resultant intervals.

Three things can be observed from Fig. 5 .

1) The position of the centre of the resultant intervals moves to the left as $\delta$ increases.

TABLE V

CC VALUES FOR PROPOSED IT2 FUZZY TOPSIS APPROACH

\begin{tabular}{|c|ccc|}
\hline & $\delta=0.10$ & $\delta=0.13$ & $\delta=0.14$ \\
\hline$A_{1}$ & {$[0.6276,0.6428]$} & {$[0.6244,0.6428]$} & {$[0.6223,0.6426]$} \\
$A_{2}$ & {$[0.7320,0.7863]$} & {$[0.7223,0.7870]$} & {$[0.7191,0.7867]$} \\
$A_{3}$ & {$[0.6820,0.7153]$} & {$[0.6760,0.7176]$} & {$[0.6740,0.7182]$} \\
\hline & $\delta=0.145$ & $\delta=0.15$ & $\delta=0.20$ \\
\hline$A_{1}$ & {$[0.6228,0.6424]$} & {$[0.6223,0.6423]$} & {$[0.6172,0.6395]$} \\
$A_{2}$ & {$[0.7160,0.7861]$} & {$[0.7160,0.7861]$} & {$[0.7010,0.7799]$} \\
$A_{3}$ & {$[0.6721,0.7187]$} & {$[0.6721,0.7187]$} & {$[0.6625,0.7192]$} \\
\hline & $\delta=0.30$ & $\delta=0.40$ & $\delta=0.50$ \\
\hline$A_{1}$ & {$[0.6077,0.6296]$} & {$[0.5995,0.6157]$} & {$[0.5925,0.6007]$} \\
$A_{2}$ & {$[0.6743,0.7570]$} & {$[0.6521,0.7276]$} & {$[0.6338,0.6979]$} \\
$A_{3}$ & {$[0.6449,0.7116]$} & {$[0.6296,0.6942]$} & {$[0.6167,0.6726]$} \\
\hline
\end{tabular}

2) The size of the intervals initially increases as $\delta$ increases, beginning to overlap across alternatives when $\delta$ reaches 0.145 - in this example.

3) However, the increasing width of the intervals is not linear with respect to increasing $\delta$, for example first growing and then shrinking again, i.e. compare the intervals for $\delta=0.10$ to $\delta=0.60$ in 0.1 increments.

As this paper, for the first time, explores the information captured in these interval-valued CC outputs, we further discuss each of the three cases in more detail below. For the first observation, in Fig. 5, it can be seen clearly that the position of the centre of the resultant intervals moves gradually to the left as $\delta$ increases from 0.10 to 0.60 . However, the ranking of alternative stays the same, but the solutions are moving further away from the theoretical ideal solution. This is an interesting observation.

Next, for the second observation in the same figure, it is clear that as $\delta$ increases from 0.10 to 0.30 , the $\mathrm{CC}$ intervals become wider. The intervals associated with $A_{2}$ and $A_{3}$ begin to intersect when $\delta$ reaches 0.145 . This result is broadly as expected in that $\delta$ indicates the amount of uncertainty in the input ratings given by the DMs, leading to increased uncertainty in the resulting recommendation produced by IT2 fuzzy TOPSIS. Whereas previous authors have used the centrepoint of the resultant intervals, it is clear that the intervals themselves provide additional information. In the real world, as uncertainty affects the inputs, the outputs need to reflect this level of uncertainty. In practice, it is likely that when the intervals overlap, then the outputs need to be interpreted with more care. The exact interpretation of the size of the output intervals and its relationship to the degree of confidence that can be placed in the overall output is something that will be further explored in future work.

Finally, the fact that the intervals do not necessarily continue to increase in size as $\delta$ increases is an interesting and novel observation. Based on the same figure, it shows that all intervals start to narrow in term of size of interval when $\delta$ reaches 0.40 and above is introduced to the MFs. Interestingly, when $\delta$ reaches 0.60 , the interval $\mathrm{CC}$ for $A 1,\left(C C_{A 1}=\right.$ $[0.5865,0.5866])$, drastically narrows and almost changes to a crisp value while the intervals $A 2,\left(C C_{A 2}=[0.6187,0.6709]\right)$ and $A 3,\left(C C_{A 3}=[0.6059,0.6508]\right)$ narrow more slowly. It is not trivial to explain these observed changes of a resulting intervals, but it may be related to relative distance among ratings given by DMs with PIS and NIS.

All of the above highlight how the traditional approach of using the mid-point of the intervals alone risks omitting important information. Further research is needed to investigate this observed findings and the interval output behaviour. As noted, considering the literature, this is the first study of IT2 fuzzy TOPSIS where the CC values adopt an interval form. By considering such intervals, more of the information that is captured in the IT2 MFs is preserved. Intuitively, evaluating the resulting information appropriate and leveraging it as part of the decision process hols substantial promise over both the 


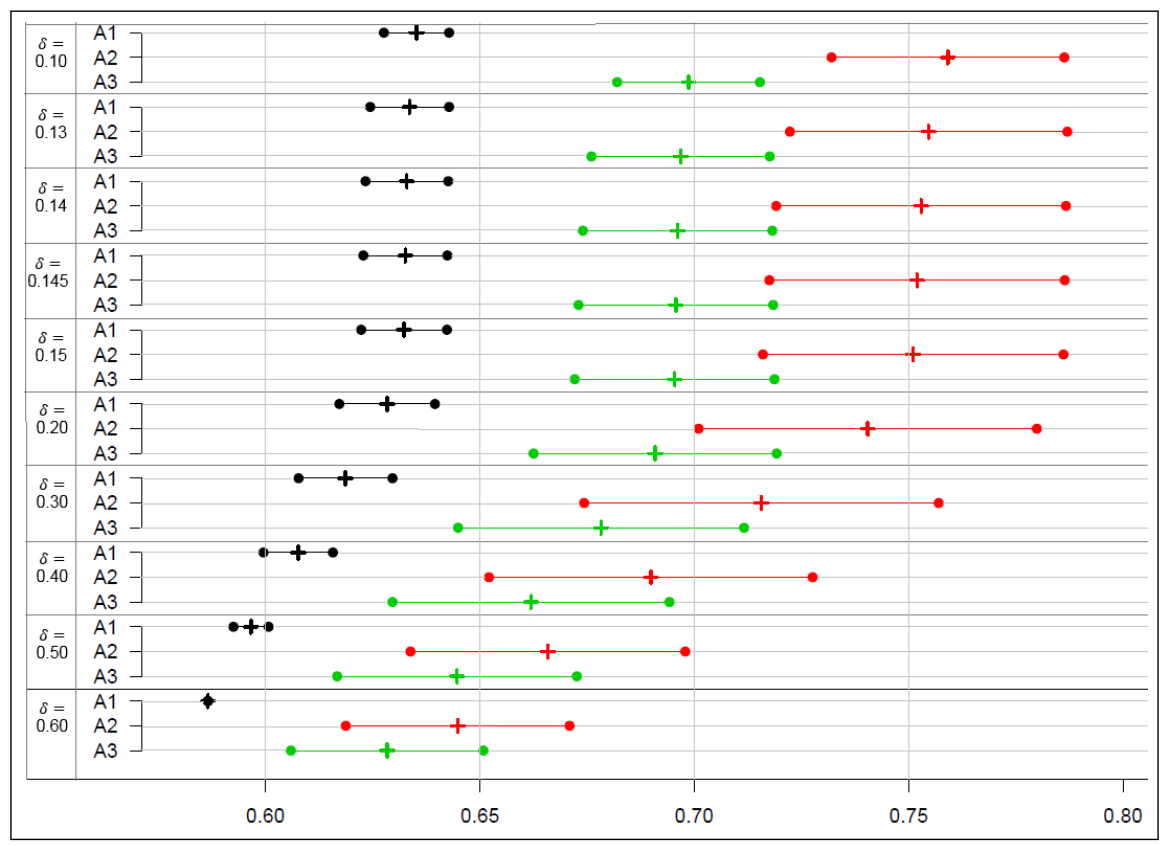

Fig. 5. The resulting closeness coefficient (CC) intervals for different values of $\delta$. The ' + ' symbol indicates the centre of each of the intervals.

T1 and the standard IT2 (preserving crisp CC outputs only) TOPSIS methods.

\section{CONCLUSiOnS}

In this paper, a new IT2 variant to TOPSIS has been proposed. A series of experiments were carried out by introducing a number of different levels of uncertainty $\delta$, to the T1 MFs associated with linguistic labels used in a standard T1 TOPSIS. The aim of the experiments was to explore how variation in the uncertainty levels in the IT2 fuzzy sets in a TOPSIS system affects the CC outputs. In particular, the proposed IT2 TOPSIS approach, for the first time, generates intervals as final outputs, which allow the reasoning about the effect of uncertainty in the inputs. The results show that there is a direct relationship between the amount of uncertainty present in the MFs and the size of the CC intervals. However, the results also highlight that this relationship is not trivial, for example, increasing uncertainty levels $\delta$ result first in increasing, and then in again shrinking output intervals.

To date, no other studies have proposed the use of intervalvalued CC values. By keeping the CC values in interval-valued form, the proposed method reduces the loss of information and maximizes the potential benefit of using IT2 FSs. In a decision-making context, this interval-valued output provides DMs with richer information, in turn allowing them to make more fine-grained and for example, potentially more cautious (if intervals are wider and overlapping) decisions. While the generic interpretation of the resulting intervals and specifically, their respective widths, is not trivial in a decision making context, the paper has established a systematic approach to linking input uncertainty to output uncertainty in TOPSIS which itself provides a rich basis for future study. In the future, we will further explore the appropriate interpretation of the intervals, while focusing in particular on real-world decision making problems.

\section{REFERENCES}

[1] Majid Behzadian, S. Khanmohammadi Otaghsara, Morteza Yazdani, and Joshua Ignatius. A state-of the-art survey of TOPSIS applications. Expert Syst. Appl., 39:13051-13069, dec 2012.

[2] Chen-Tung Chen. Extensions of the TOPSIS for group decision-making under fuzzy environment. Fuzzy Sets Syst., 114(1):1-9, aug 2000.

[3] Shyi Ming Chen and Li Wei Lee. Fuzzy multiple attributes group decision-making based on the interval type-2 TOPSIS method. Expert Syst. Appl., 37(4):2790-2798, 2010.

[4] Ludmila Dymova, Pavel Sevastjanov, and Anna Tikhonenko. An interval type-2 fuzzy extension of the TOPSIS method using alpha cuts. Knowledge-Based Syst., 83:116-127, 2015.

[5] Irfan Ertugrul and Nilsen Karakasoglu. Comparison of fuzzy AHP and fuzzy TOPSIS methods for facility location selection. Int. J. Adv. Manuf. Technol., 39:783-795, 2007.

[6] Jonathan M. Garibaldi and Turhan Ozen. Uncertain fuzzy reasoning: A case study in modelling expert decision making. IEEE Trans. Fuzzy Syst., 15:16-30, 2007.

[7] Ching-Lai Hwang and Kwangsun Yoon. Multiple Attribute Decision Making : Methods and Application- A State of the Art Survey. Springer Berlin Heidelberg New York, lecture no edition, 1981.

[8] Mesut Kilic and hsan Kaya. Investment project evaluation by a decision making methodology based on type-2 fuzzy sets. Appl. Soft Comput., 27:399-410, 2015.

[9] Francisco Rodrigues Lima Junior, Lauro Osiro, and Luiz Cesar Ribeiro Carpinetti. A comparison between Fuzzy AHP and Fuzzy TOPSIS methods to supplier selection. Appl. Soft Comput., 21:194-209, 2014.

[10] Elissa Nadia Madi, Jonathan M Garibaldi, and Christian Wagner. An Exploration of Issues and Limitations in Current Methods of TOPSIS and Fuzzy TOPSIS. In IEEE Int. Conf. Fuzzy Syst., pages 2098-2105, 2016.

[11] F Ghaemi Nasab. Extension of TOPSIS for Group Decision-Making Based on the Type-2 Fuzzy Positive and Negative Ideal Solutions. Int. J. Ind. Math., 2(3):199-213, 2010.

[12] Xiuzhi Sang and Xinwang Liu. An analytical solution to the TOPSIS model with interval type-2 fuzzy sets. Soft Comput., 2015. 